\title{
The HF-CGM study: An analysis of cardiogoniometric axes in patients with cardiac resynchronization therapy
}

\author{
Oliver I Brown* $\uparrow$, Theodora Nikolaidou $\uparrow$, Gowan Beddoes, Angela Hoye, Andrew L Clark
}

\begin{abstract}
Goal: The HF-CGM is a proof-of-principle study to investigate whether cardiogoniometry (CGM), a threedimensional electrocardiographic method, can differentiate between pacing modes in patients with cardiac resynchronisation therapy (CRT). Methods: At a tertiary cardiology centre, CGM recordings were performed using four pacing modes: no pacing; right ventricular (RV) pacing; left ventricular (LV) pacing and biventricular (BIV) pacing. Three orthogonal CGM planes orientated to the long axis (XY), the frontal plane (YZ) and the short axis $(\mathrm{XZ})$ of the heart were constructed, and the direction of the QRS axis was calculated for each pacing mode in each plane. During BIV pacing, the direction of CGM QRS axis was compared between patients with optimal and non-optimal 12-lead pacing variables. Results: Twenty-two participants (aged 71.5 \pm 10.8 ; $\mathbf{7 7 . 3 \%}$ male, LVEF $29 \pm 7 \%$ ) were consecutively recruited. Only QRS axis measured in the XY plane could significantly distinguish between all three pacing modes vs. no pacing. Mean QRS axis in the XY plane with pacing off and during RV pacing was leftwards and basal; $L V$ pacing was apical; and BIV pacing was rightwards and basal. There was a statistically significant difference in the direction of QRS axis between patients with optimal vs. nonoptimal paced QRS morphology in the XY plane (rightwards and basal vs. inconsistent). Significance: CGM recorded in the XY plane can accurately detect differences between ventricular pacing sites. It may also be able to identify patients with a CRT device in situ who have optimal response.
\end{abstract}

Index Terms - Cardiac axis, Cardiac Resynchronization therapy, Cardiogoniometry, Heart Failure

\section{INTRODUCTION}

ardiac resynchronization therapy (CRT) improves both the symptoms and the prognosis of patients with chronic heart failure [1], and is indicated in people with heart failure and left bundle branch block (LBBB).

Unfortunately, approximately $25 \%$ of patients do not gain significant clinical benefit with CRT [2]. Such patients are termed "non-responders", and lack of response is typically measured as a failure to improve exercise capacity with CRT, or a failure of left ventricular function to improve on echocardiography. Many methods have been proposed to improve response to CRT, but no specific technique has been shown to be superior to another and the long term clinical benefit of each technique is unclear [3].

Cardiogoniometry (CGM) is a method of vectorcardiographic (VCG) assessment [4][5]. The Cardiologic Explorer (Enverdis GmBH, Jena, Germany) is the only commercially available device. It uses five electrodes arranged to make a recording from three virtual bipolar leads (see Fig. I). Three orthogonal planes can be constructed from the bipolar leads and are approximately orientated to the long axis (XY) of the heart, the frontal plane (YZ) and the short axis (XZ) of the heart (see Fig. II). A heart vector can be plotted in each plane over time, resulting in vector loops in three dimensions for the $\mathrm{P}, \mathrm{QRS}$ and $\mathrm{T}$ waves. The introduction of quadripolar leads and multipoint pacing have made it possible to alter CRT LV pacing site at follow-up to achieve better response. This optimisation is routinely done using ECG. Similar to ECG, CGM is a noninvasive, quick method that can be used at the bedside. However, CGM has greater three-dimensional resolution and CGM-guided optimisation of CRT may be superior to that using ECG, leading to better patient outcomes.

Previous work using CGM has been limited to investigating its diagnostic performance in patients with suspected coronary artery disease [6][7]. However, the effect of CRT on QRS axis has not been formally described. Interestingly, recent work with other methods of VCG has indicated that VCG can help improve response to CRT [8-10]. Furthermore, the direction of cardiac electrical activity after CRT implantation also predicts improvement in left ventricular ejection fraction (LVEF) [11] [12].

We conducted a pilot study (HF-CGM) to assess the ability
The Manuscript was received July 20th 2017, and revised on the $3^{\text {rd }}$ of October 2017. This work was supported by This study was funded by a grant provided by The Hull \& East Riding Cardiac Trust Fund (Kingston upon Hull, United Kingdom). O I Brown was supported by a Wolfson Intercalated Award, awarded on the behalf by the Royal College of Physicians (London, United Kingdom). There are no relationships with industry to declare.

T Nikolaidou, A Hoye and A L Clark are with the department of Academic Cardiology, University of Hull, Kingston upon Hull, East Yorkshire, United Kingdom. G Beddoes is with the Department of Cardiology, Hull and East (C) 20xx IEEE. Personal use of this material is permitted. Permission from IEEE must be obtained for all other uses, in any current or future media, including reprinting/republishing this material for advertising or promotional purposes, creating new collective works, for resale or redistribution to servers or lists, or reuse of any copyrighted component of this work in other works.

Yorkshire Hospitals NHS trust, Kingston upon Hull, East Yorkshire, United Kingdom. *O I Brown is with the department of Academic Cardiology, University of Hull, Kingston upon Hull, East Yorkshire, United Kingdom (correspondence email: oliver.brown@hyms.ac.uk). † - O I Brown and T Nikolaidou share co-first authorship on this manuscript.

Copyright (c) 2016 IEEE. Personal use of this material is permitted. However, permission to use this material for any other purposes must be obtained from the IEEE by sending an email to pubs-permissions@ ieee.org 
of CGM derived QRS axis to detect changes in CRT pacing site.

\section{Methodology}

\section{A. Study participants}

Twenty-two patients undergoing routine CRT device checks were recruited between November 2015 and November 2016. During implantation the right ventricular lead was placed in either the apical or low septal position. Positioning of the left ventricular lead was guided by coronary sinus venography to the lateral or posterolateral region. In one case, this was not technically possible and the lead was deployed in the great cardiac vein. For inclusion, patients had to be aged 18 or over, have a functioning CRT device implanted and be able to provide informed written consent. Patients who were pacemaker dependent (i.e. had no intrinsic electrical activity) and non-English speakers were excluded from the study.

\section{B. Ethics}

The study protocol along with all other documentation was approved by the regional ethics committee (15/NW/0479 and 16/YH/0185). The study was registered on http://www.clinicaltrials.gov/, unique identifier: NCT02803879 and NCT02748876. We conducted our study in compliance with the principles of the Declaration of Helsinki.

\section{Study Protocol}

Four CGM electrodes were placed on the patient's thorax, with a fifth CGM electrode placed on the patient's left thigh to act as a grounding electrode. Eleven patient underwent the following sequential CGM recordings whilst lying as still as possible whilst the recording was in progress: (1) biventricular pacing (BIV) with no changes in device settings; (2) pacing via the RV lead alone; (3) pacing via the LV lead alone; and (4) both RV and LV leads turned off. Another eleven patients had $\mathrm{BiV}$ and off pacing recordings only. All recording were obtained with patients breath-holding at peak inspiration. For paced rhythms, traces were obtained at a minimum paced rate of $80 \mathrm{bpm}$ (or until intrinsic electrical activity had been overcome) so the recordings taken were a reflection of paced cardiac axis and not intrinsic electrical activity. Atrioventricular conduction was intrinsic or set to default delay settings.

12-lead ECGs were also recorded to allow comparisons of mean frontal cardiac axis calculated by both methods. The limb electrodes for the 12-lead ECG were placed on the patient's wrists and ankles, precordial electrodes were placed in Wilson positions $\mathrm{V}_{1}$ to $\mathrm{V}_{6}$ [13]. Finally, patients had their CRT settings restored (or optimised using conventional methods if clinically indicated).

\section{Data analysis}

CGM data were recorded using the Patient Explorer software version 2.1 [Enverdis, Jena, Germany]. For each of the CGM recordings, the mean QRS axis (in degrees) was calculated as follows: the net deflection of the QRS complex $(\mathrm{mV})$ was measured for each of the $\mathrm{X}, \mathrm{Y}$ and $\mathrm{Z}$ axes to produce orthogonal coordinates. Polar angles for the XY, YZ, and XZ planes were calculated using formulae previously described by Sanz et al in 1983 [4] (Appendix 1.1 in the supplementary material). The mean frontal QRS axis was calculated from the 12 lead ECG. The net deflection of the QRS complex measured in the plane defined by the orthogonal leads I and aVF was used to produce coordinates, which were subsequently transformed to polar angles by trigonometry (Appendix 1.2 in the supplementary material). This method is validated for calculating the cardiac axis [14].

\section{A Placement of electrodes}
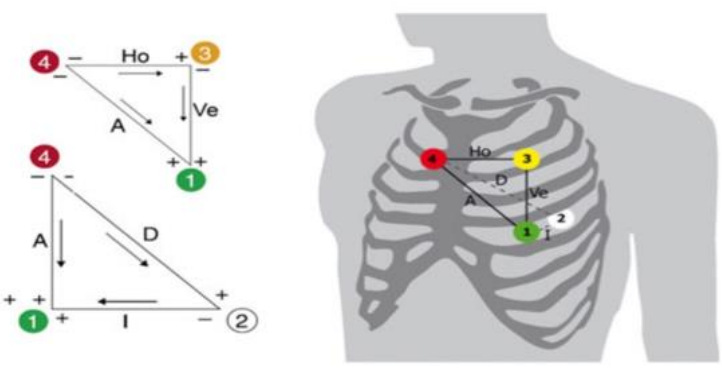

B Calculation of three orthogonal

B leads XYZ and 3D projection planes
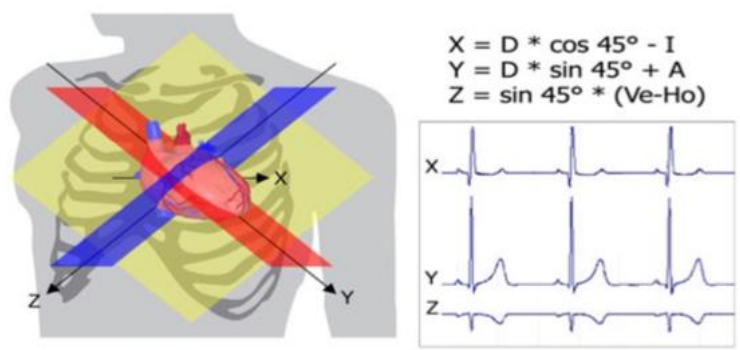

C Calculation of vector loops

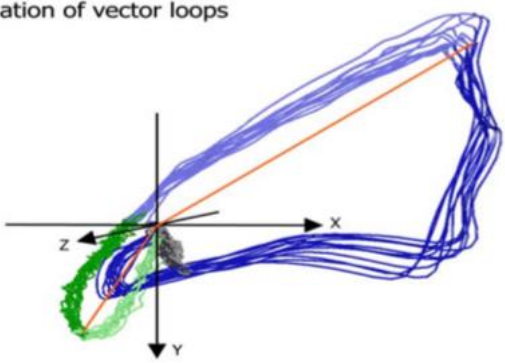

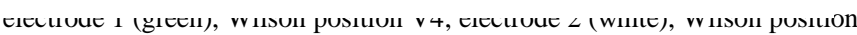
V8; electrode 3 (yellow), directly superior to electrode 1 at a distance 0.7 times the distance between electrode 1 and 2; electrode 4 (red), directly right of electrode 3 at a distance the same as between electrode 1 and 3 . The following leads are defined by the following electrodes: Anterior (A) by electrode $4 \rightarrow 1$; Horizontal (Ho) by electrode $4 \rightarrow 3$; Vertical (Ve) by electrode $3 \rightarrow 1$; Inferior by electrode $2 \rightarrow 1$ and Diagonal (D) by electrode $4 \rightarrow 2$ (see figure $2 \mathrm{~A}$ ). Triangles left of the thorax show the direction of the aforementioned leads. B: Showing the orientation of orthogonal axes X, Y and $\mathrm{Z}$ in the thorax (left panel) and trigonometric equations defining their formation (right panel); C: Showing the formation of vector loops by plotting of the heart vector at every millisecond for the P (grey), QRS (blue) and T waves (green), with maximum vectors for the $\mathrm{P}$ and QRS loop (orange lines) being shown. Reproduced from Schupbach et al [6]. 
The CGM YZ plane is claimed to be equivalent to the frontal plane calculated by the ECG rotated by $-45^{\circ}$, and so we subtracted $45^{\circ}$ from the axis calculated from the conventional ECG to allow direct comparison with the axis calculated from CGM [4].

\section{E. Statistical analysis}

IBM SPSS Statistics for Macintosh, Version 23.0 was used for statistical analysis of baseline clinical characteristics. Descriptive statistics were used to summarise the data. Baseline continuous variables are expressed as mean $\pm \mathrm{SD}$, categorical data are expressed as numbers/percentages. Statistical analysis for the axis data was performed using RStudio Version 0.99.491 (RStudio Inc, Boston, USA). Values for the cardiac axis were first transformed from linear to circular format, with the scale in degrees going from $0 \rightarrow 180 /-180 \rightarrow 0$. The mean and $95 \%$ confidence interval (based on a Von Mises distribution) [15] for each CRT pacing site in each plane was calculated. Differences between pacing sites were assessed with the non-parametric Moore's test for paired circular data, with RV pacing, LV pacing and biventricular pacing compared to no pacing. The equivalence of the frontal CGM plane to the conventional ECG plane was assessed by visual comparison of scatterplots. A secondary analysis was performed based on whether patients had satisfied the 'optimal' QRS morphology defined by Bode et al [11] (R/S ratio $\geq 1$ in $\mathrm{V} 1$ and/or $\mathrm{R} / \mathrm{S}$ ratio $\leq 1$ in lead $\mathrm{I})$ on their initial ECG with CRT. Statistical differences between 'optimal' and 'non-optimal' groups were assessed with Watson's non-parametric test and visual comparison of circular scatter plots. Statistical significance was pre-defined as $\mathrm{p} \leq 0.05$.

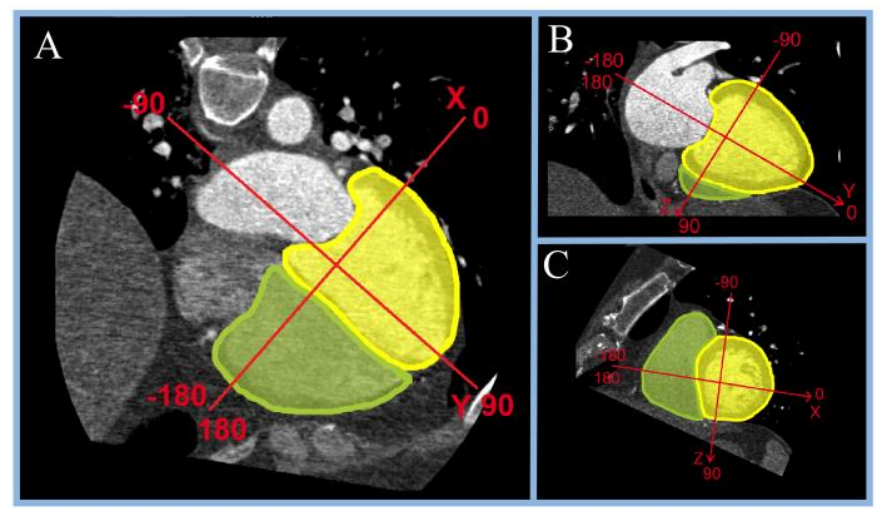

\section{RESULTS}

Fig. 2. Cardiac CT images of one patient in the same approximate sections as the CGM planes -XY plane (panel A), YZ plane (panel B), $\mathrm{XZ}$ plane (panel C). Green shading represents the right ventricle; yellow shading represents the left ventricle. Images used with permission.

Baseline characteristics for study participants are shown in Table.

TABLE I

BASELINE CHARACTERISTICS OF STUDY PARTICIPANTS Demographics

\begin{tabular}{ll}
\hline Past medical history $(\%)$ & \\
\hline Myocardial infarction & $9(40.9)$ \\
Percutaneous coronary intervention & $11(50.0)$ \\
Atrial fibrillation & $5(22.7)$ \\
Stroke/ Transient ischemic attack & $5(22.7)$ \\
Chronic kidney disease & $13(59.1)$ \\
Diabetes mellitus & $12(54.5)$ \\
Hypertension & $12(54.5)$ \\
Hypercholesterolaemia & $14(63.6)$ \\
Smoking (Never/Ex/Current) & $7(31.8 / 14(63.6) / 1(4.5)$ \\
\hline Medications (\%) & \\
\hline Aspirin & $8(36.4)$ \\
Clopidogrel & $4(18.2)$ \\
ACEi & $12(54.5)$ \\
ARB & $8(36.4)$ \\
$\beta$-blocker & $20(90.1)$ \\
MRA & $13(59.1)$ \\
Loop diuretic & $19(86.4)$ \\
Digoxin & $4(18.2)$
\end{tabular}

Blood results (\%)

Haemoglobin, g/L

Sodium, $\mathrm{mmol} / \mathrm{L}$

Potassium, $\mathrm{mmol} / \mathrm{L}$

Chloride, $\mathrm{mmol} / \mathrm{L}$

Urea, $\mathrm{mmol} / \mathrm{L}$

Creatinine, $\mu \mathrm{mol} / \mathrm{L}$

NT proBNP, ng/L

Continuous data are expressed as mean and standard deviation (SD) Abbreviations: angiotensin converting enzyme inhibitor (ACEi), angiotensin receptor blocker (ARB), atrioventricular (AV), Intraventricular (VV), left bundle branch block (LBBB), left ventricle (LV), mineralocorticoid receptor antagonist (MRA)

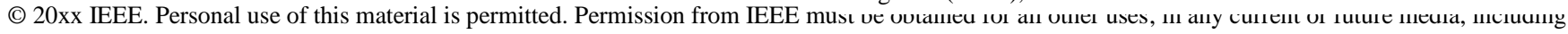
reprinting/republishing this material for advertising or promotional purposes, creating new collective works, for resale or redistribution to servers or lists, or reuse of any copyrighted component of this work in other works. 


\section{A. Direction of cardiac axis by pacing settings}

Mean angles and their 95\% confidence interval for each device setting in each plane are shown in Table II. Biventricular pacing led to a very wide range of readings in all three planes. The narrowest ranges for axis were in the XY plane, and only in the $\mathrm{XY}$ plane were there significant differences in axis between all three different pacing modes compared to no pacing (Table III). There was a significant difference in axis in the XZ plane between no pacing and biventricular pacing, but not in the $\mathrm{YZ}$

plane.

TABLE II

MEAN DIRECTION AND 95\% CONFIDENCE INTERVALS OF CARDIAC RESYNCHRONIZATION DEVICE SETTINGS IN EACH CARDIOGONIOMETRY PLANE.

\begin{tabular}{llll}
\hline \hline & XY plane $(\mathrm{CI})$ & \multicolumn{1}{c}{ YZ plane $(\mathrm{CI})$} & XZ plane $(\mathrm{CI})$ \\
\hline No pacing & $-21^{\circ}\left(-28^{\circ} \rightarrow 13^{\circ}\right)$ & $-156^{\circ}\left(-173^{\circ} \rightarrow-138^{\circ}\right)$ & $-7^{\circ}\left(-24^{\circ} \rightarrow 11^{\circ}\right)$ \\
RV pacing & $-51^{\circ}\left(-61^{\circ} \rightarrow-40^{\circ}\right)$ & $-175^{\circ}\left(174^{\circ} \rightarrow-164^{\circ}\right)$ & $-8^{\circ}\left(-20^{\circ} \rightarrow 3^{\circ}\right)$ \\
LV pacing & $169^{\circ}\left(126^{\circ} \rightarrow-147^{\circ}\right)$ & $16^{\circ}\left(-162^{\circ} \rightarrow 178^{\circ}\right)$ & $149^{\circ}\left(-1^{\circ} \rightarrow-62^{\circ}\right)$ \\
BIV pacing & $-104^{\circ}\left(-147^{\circ} \rightarrow-61^{\circ}\right)$ & $-170^{\circ}\left(150^{\circ} \rightarrow-138^{\circ}\right)$ & $-78^{\circ}\left(162^{\circ} \rightarrow 42^{\circ}\right)$ \\
\hline
\end{tabular}

Continuous data are expressed as mean and $95 \%$ confidence interval.

Abbreviations: biventricular pacing (BIV); confidence interval (CI); left ventricular pacing $(\mathrm{LV})$; right ventricular pacing $(\mathrm{RV})$.

TABLE III

P VALUES CALCULATED FOR MEAN DIFFERENCES FOR CARDIAC AXIS BETWEEN DEVICE SETTINGS; NO PACING VS. RIGHT VENTRICULAR, LEFT VENTRICULAR AND BIVENTRICULAR PACING FOR EACH CARDIOGONIOMETRY PLANE.

\begin{tabular}{lccc}
\hline \hline & XY plane & YZ plane & XZ plane \\
\hline None vs. RV & 0.0001 & 0.410 & 0.356 \\
None vs. LV & 0.0001 & 0.005 & 0.010 \\
None vs. BIV & 0.0001 & 0.118 & 0.001
\end{tabular}

Abbreviations: biventricular pacing (BIV); left ventricular pacing (LV); right ventricular pacing $(\mathrm{RV})$.

\section{B. Mean QRS axis: ECG plane vs. CGM YZ plane}

There was little agreement in the direction of the cardiac axis between the YZ CGM plane and conventional ECG plane (Fig. 3).

\section{C. 'Optimal' $Q R S$ axis vs. non-optimal $Q R S$ axis morphology}

Fig. 4. shows scatter plots for patients with optimal vs. nonoptimal paced QRS morphology in each CGM plane. In the XY plane, the axis of patients with optimal paced QRS morphology was mostly directed between $-90^{\circ}$ to $-180^{\circ}$, towards the right basal side of the heart, whereas there was no consistency in the direction of axis in patients with a non-optimal paced QRS morphology. This difference in axis direction was statistically significant $(\mathrm{p}=0.005)$. In the other two planes, there was a large overlap in the direction of axis for participants with an optimal paced QRS morphology and non-optimal paced QRS morphology, with no consistent pattern in axis.

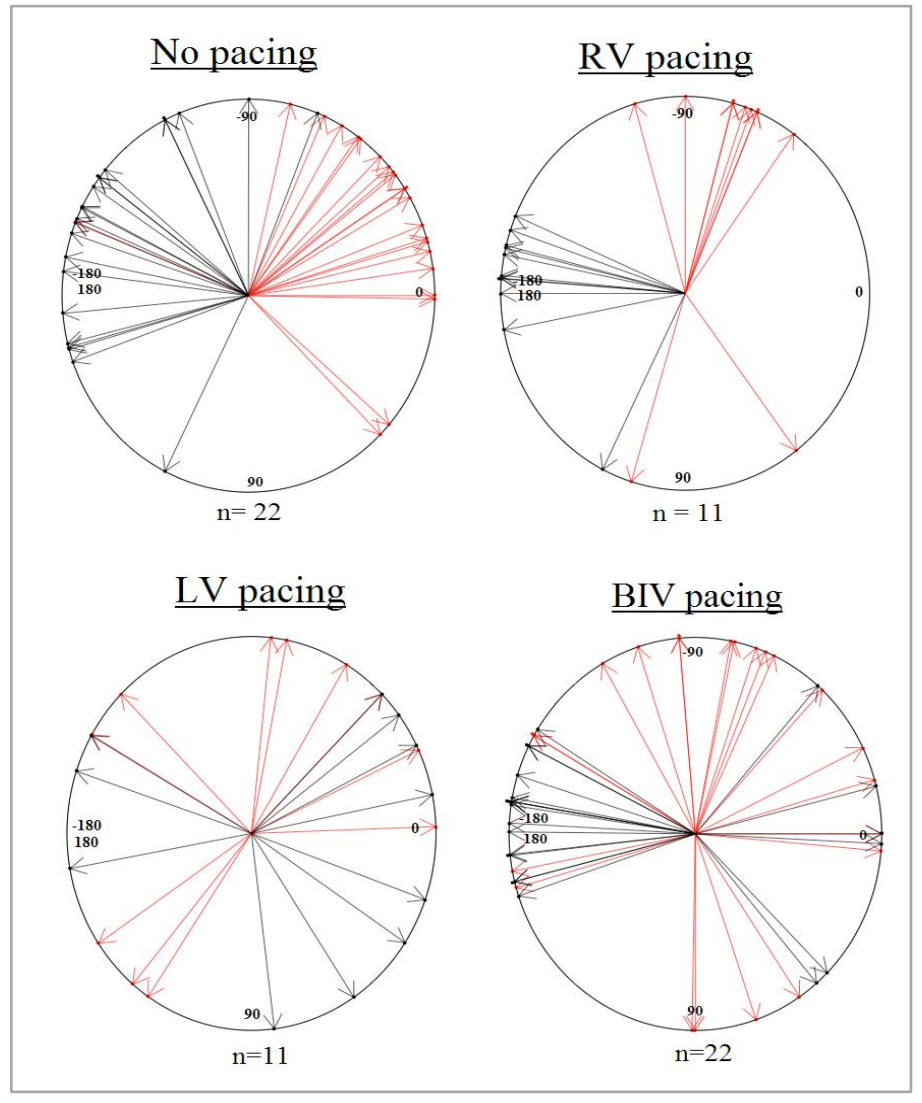

Fig. 3. (above) Circular scatter plot demonstrating individual study participants' cardiac axes with no pacing, right ventricular (RV) pacing, left ventricular (LV) pacing, and biventricular (BIV) pacing. Black arrows represent cardiac axis on CGM YZ plane, red arrows represent cardiac axis on 12 lead ECG frontal plane.

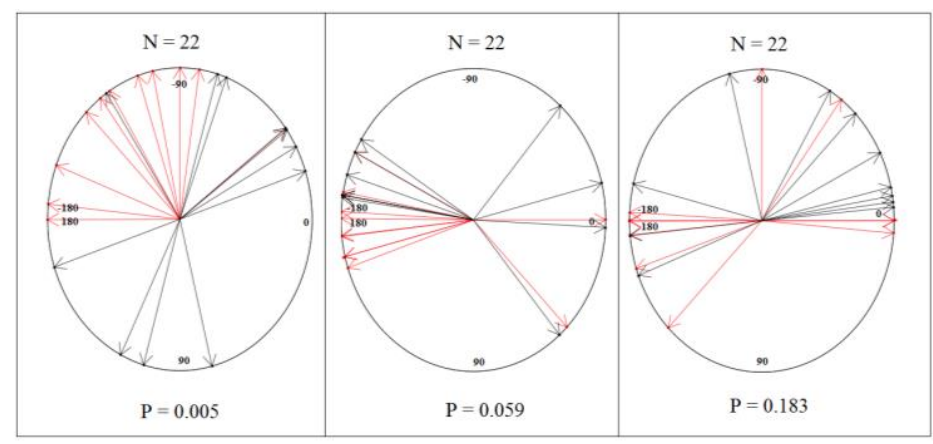

Fig. 4. Circular scatter plots showing the direction of cardiac axis of patients with 'optimal' paced QRS morphology (red arrows) vs. 'non-optimal' paced QRS morphology (black arrows) in each CGM plane: XY plane (left), YZ plane (centre), XZ plane (right). P value for statistical difference between optimal and non-optimal paced QRS morphology shown below each plot.

\section{DISCUSSION}

We have found that different CRT device settings lead to differences in CGM recordings, and that the most consistent patterns are seen with recordings in the XY plane. We have also shown that the electrical axis recorded in the $\mathrm{YZ}$ plane using CGM is not the same as the mean frontal QRS axis recorded in the frontal plane of the ECG as previously thought, even after rotating by $45^{\circ}$. Finally, we have demonstrated that the XY plane can identify the direction of electrical activity which is

(C) 20xx IEEE. Personal use of this material is permitted. Permission from IEEE must be obtained for all other uses, in any current or future media, including reprinting/republishing this material for advertising or promotional purposes, creating new collective works, for resale or redistribution to servers or lists, or reuse of any copyrighted component of this work in other works. 
associated with an "optimal" paced QRS morphology.

In our study, the direction of axis in the XY plane corresponded to what might be expected on theoretical grounds. $\mathrm{RV}$ pacing causes the heart to depolarize from the apex of the RV and depolarization therefore moves basally. We found that QRS axis during RV pacing was directed posteriorly and towards the left base of the heart. LV pacing causes the heart to depolarize from the $\mathrm{LV}$ free wall, and therefore the depolarization is directed anteriorly towards the RV: the pattern we found. During BIV pacing, the direction of electrical activity varies depending on the timing delays and location of the LV and RV leads. Nevertheless, the overall cardiac depolarization during BIV pacing should be directed basally. We found that during BIV pacing the QRS axis was directed towards the right basal side of the heart (with some variation in overall direction).

The XY plane, oriented along the long axis of the heart, includes large parts of both RV and LV, which may explain why recordings in this plane were significantly different between each of the pacing modes. Although the YZ (frontal) plane is aligned to the long axis of the heart to some degree, the section it takes through the heart predominately contains LV, and therefore is a poor representation of biventricular electrical activity. The XZ plane contains similar amounts of both RV and $\mathrm{LV}$ is orientated to the short axis of the ventricles and therefore does not reflect depolarization from apex to base, but rather relates to depolarization from the endocardium to epicardium.

Depolarization in the XY plane also better discriminated between "optimal" CRT delivery and sub-optimal. Of course, the definition of "optimal" is to some degree arbitrary and based on analysis of 12 lead ECGs. It might be that CGM provides additional information which may prove more helpful, especially if an 'optimal' range of paced QRS axis can be determined.

The trigonometric construction of the CGM YZ and the frontal ECG planes both take a coronal slice through the heart: and, not surprisingly, previous reports have suggested that the two are equivalent [4]. However, we have demonstrated that the two are not the same. A possible explanation is that the reference points for the CGM and the 12-lead ECG are different as CGM does not use Wilson's central terminal. Furthermore, the construction of the CGM YZ plane is fundamentally different: it uses information from an electrode on the back (electrode 2) which is not used in the 12-lead ECG.

Whether CGM has anything to offer in clinical practice needs to be tested further. Does pacing from different points on a multipolar LV lead alter the CGM readings? One potential study would be to relate CGM findings to clinical response to CRT in a larger sample of patients .using, say, a 6-minute walk test, a disease-specific quality of life score and left ventricular end systolic volume. Is there a relation between CGM variables and "response"? A randomised study might then explore whether there is any clinical benefit to manipulating pacing sequences based on their effect on CGM variables.

\section{A - Study Limitations}

The sample size of the study was small. However, the study was designed as a pilot and the fact we were able to demonstrate statistically significant differences suggests CGM may have a role in assessing CRT delivery. Cardiac axes for both CGM and ECG were calculated by hand which could bring a degree of human error. Participants had different CRT devices with different atrioventricular and interventricular programmed settings and the devices and had been implanted at different time-points. In particular we did not standardize the paced/sensed atrioventricular delay, which has previously been shown to affect axis direction [9]. In addition, there may have been differences in LV pacing site depending on each individual's coronary venous anatomy. We did not have data on the extent and localization of myocardial scar, which may have impacted on axes measurements. In addition, native and paced electrical axes may change over time.

We did not measure other cardiogoniometric variables such as QRS area within the vector loops for different pacing settings which might provide useful information. QRS area assessed by VCG predicts response to CRT [8][16]. In addition to this, we only looked at direction of the axis of ventricular depolarization, and information may be gained from the axes of atrial depolarization and ventricular repolarization [17].

\section{CONCLUSION}

CGM can detect differences between ventricular pacing sites. It is able to identify patients with a paced QRS morphology associated with improvement in clinical endpoints. CGM should further be evaluated to explore whether CGM-derived axes might help guide CRT lead placement and pacing timing intervals to improve patient outcomes.

\section{ACKNOWLEDGMENT}

The authors would like to thank the Hull and East Riding Cardiac Trust for their support of the study. We would also like to thank Jan Knobeloch from Enverdis for technical advice.

\section{REFERENCES}

[1] J. G. F. Cleland et al, "The Effect of Cardiac Resynchronization on Morbidity and Mortality in Heart Failure," N Eng J Med, vol. 352, no. 15, pp. 1539-1549, Apr 2005.

[2] A. Auricchio, F.W. Prinzen, "Non-Responders to Cardiac Resynchronization Therapy”, Circ J, vol. 75, no.3, pp. 521527, Mar 2011.

[3] F.A. Cuoco, M.R. Gold, "Optimization of cardiac resynchronization therapy: Importance of programmed parameters," J Cardiovasc Electrophysiol, vol. 23, no.1, pp. 110-118, Jan 2012.

[4] E. Sanz, J. P. Steger, W.Thie, "Cardiogoniometry", Clin Cardiol, vol. 6, pp. 199-206, May 1983.

[5] H. Saner et al, "Cardiogoniometry: a new noninvasive method for detection of ischemic heart disease," Clin Cardiol, vol.6, pp. 207-206, May 1983.

[6] W. M. M. Schüpbach et al, "Non-invasive diagnosis of

(c) 20xx IEEE. Personal use of this material is permitted. Permission from IEEE must be obtained for all other uses, in any current or future media, including

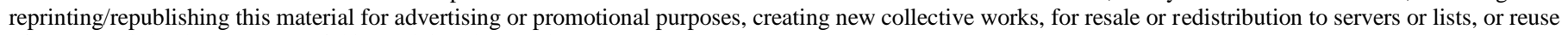
of any copyrighted component of this work in other works. 
coronary artery disease using cardiogoniometry performed at rest," Swiss Med Wkly, vol.138, no. 15, pp. 230-238, Apr 2008.

[7] R. Tölg et al, "Cardiogoniometry as a diagnostic tool in patients with acute coronary syndromes: results of the CGM@ACS trial," Clin Res Cardiol, vol.101, no. 9, pp. 727736, Sept 2012.

[8] C. J. M. van Deursen et al, "Vectorcardiography for optimization of stimulation intervals in cardiac resynchronization therapy," J Cardiovasc Transl Res, vol. 8, no. 2, pp. 128-137, Mar 2015.

[9] C. J. M. van Deursen et al, "Vectorcardiography as a tool for easy optimization of cardiac resynchronization therapy in canine left bundle branch block hearts," Circ Arrhythm Electrophysiol, vol. 5, no. 3, pp. 544-552, Jun 2012.

[10] M. Mafi Rad et al, "Vectorcardiographic QRS area identifies delayed left ventricular lateral wall activation determined by electroanatomic mapping in candidates for cardiac resynchronization therapy," Heart Rhythm, vol. 13, no. 1, pp. 217-225, Jan 2016.

[11] W.D. Bode et al, "Prominent R wave in ECG lead V1 predicts improvement of left ventricular ejection fraction after cardiac resynchronisation therapy in patients with or without left bundle branch block," Heart Rhythm, vol. 12, no. 10, pp. 2141-2147, Oct 2015.

[12] E. Coverstone et al, "The postimplantation electrocardiogram predicts clinical response to cardiac resynchronization therapy", Pacing Clin Electrophysiol, vol. 38, no. 5, pp. 572580, May 2015.

[13] A.R. Barnes et al, "Standardization of precordial leads. Supplementary report," Am Heart J, vol. 15, no. 2, pp. 235239, Feb 1938.

[14] D.H. Spodick, M. Frisella, S. Apiyassawat, "QRS Axis Validation in Clinical Electrocardiography," Am J Cardiol, vol.101, no. 2, pp. 268-269, Jan 2008.

[15] J. Zar , "Circular Distributions: Hypothesis Testing" in Biostatistical analysis, $5^{\text {th }}$ ed. Upper Saddle River, USA: Prentice-hall Inc, 2010, ch. 27, pp. 665-713.

[16] C. J. M. Van Deursen et al, "Vectorcardiographic QRS area as a novel predictor of response to cardiac resynchronization therapy," J Electrocardiol, vol. 48, no.1, pp.45-52, 2015.

[17] E. Engels et al, "T-wave area predicts response to cardiac resynchronisation therapy in patients with left bundle branch block," J Cardiovasc Electrophysiol, vol. 26, no. 2, pp. 176 183, Feb 2015. 